\title{
DHEA supplementation improves endometrial HOXA-10 mRNA expression in poor responders
}

\author{
Önder Çelik ${ }^{1}$, Mustafa Acet ${ }^{2}$, Aytaç İmren ${ }^{3}$, Nilüfer Çelik ${ }^{4}$, Aynur Erşahinn ${ }^{5}$, Lebriz Hale Aktun², Barış Otlu6, \\ Sudenaz Çelik ${ }^{7}$, Eray Çalışkan ${ }^{8}$, Cihat Ünlü 9 \\ 1 Private Clinic, Obstetrics and Gynecology, Uşak, Turkey \\ 2Department of Obstetrics and Gynecology, Medipol University Faculty of Medicine, İstanbul, Turkey \\ ${ }^{3}$ Clinic of Obstetric and Gynecology, Medical Park Hospital, Uşak, Turkey \\ ${ }^{4}$ Clinic of Biochemistry, Behçet Uz Children's Hospital, İzmir, Turkey \\ 5Department of Obstetrics and Gynecology, Bahçeşehir University Faculty of Medicine, İstanbul, Turkey \\ 6Department of Medical Microbiology, İnönü University Faculty of Medicine, Malatya, Turkey \\ ${ }^{7}$ Kent College Güzelbahçe High School, İzmir, Turkey \\ 8Department of Obstetrics and Gynecology, Bahçeşehir University Faculty of Medicine, Kocaeli, Turkey \\ 9 Department of Obstetrics and Gynecology, Acıbadem University Faculty of Medicine, İstanbul, Turkey
}

\section{Abstract}

Objective: The study was planned to investigate whether DHEA supplementation had an impact on endometrial receptivity in women who were poor responders (POR).

Material and Methods: Twenty-eight POR women who were undergoing hysteroscopy and five fertile control subjects were included. The POR women were equally subdivided into two separate groups as patients who were currently using DHEA and those who were not. Endometrial samples of the subjects were obtained during hysteroscopy at the late follicular phase. Expression levels of endometrial HOXA-10, HOXA-11, and LIF mRNA were measured with the using real-time polymerase chain reaction. Spontaneous clinical pregnancy rates were also noted.

Results: Compared with POR women who were not given DHEA, upregulated endometrial HOXA-10 (7.33-fold) and HOXA-11 (2.39-fold) mRNA expression were detected in POR women on DHEA. The increase in HOXA-10 mRNA was significant $(\mathrm{p}<0.03)$. The fold increase in HOXA-11 mRNA was found as 2.39, which indicated a positive upregulation. However, this fold increment was insignificant ( $p<0.45)$. An insignificant increase in spontaneous clinical pregnancy rates in POR women on DHEA (53.3\%) was observed compared with POR women who were not given DHEA (43.8\%).

Conclusion: Oral DHEA supplementation in POR upregulates endometrial HOXA-10 mRNA expression, which is known to positively modulate endometrial receptivity. (J Turk Ger Gynecol Assoc 2017; 18: 160-6)

Keywords: Endometrium, DHEA, homeobox genes, poor responder

Received: 2 July, 2017 Accepted: 12 October, 2017

\section{Introduction}

A fall in androgens, their metabolites, and DHEA decrease progressively during reproductive period (1-3). DHEA is a critical substrate for sex steroid production in aging women (4). A recent review reported that ovarian synthesis of DHEA decreases with age (5). A little more than half of DHEA is of adrenal origin, the remaining amount is released from the ovary
(6). Similarly, the pool of androgens including DHEA decreases gradually with age $(7,8)$. Local endometrial androgen levels also decrease due to a remarkable decline in the synthesis of adrenal androgens. All these could be responsible for the decline in reproductive performance in aging women (7). The decrease in ovarian reserve is not only a reality in older women, it also might occur in young women with infertility 
$(1,2)$. DHEA looks like a breakthrough therapeutic medication in improving ovarian responses in poor-responder (POR) patients. Despite the common use of DHEA as a supplement in POR, the exact mechanism of DHEA action on reproductive events remains speculative. To date, information related to the impact of DHEA on reproductive outcome has largely focused on the potential for the count of retrieved oocytes. Concordantly, androgens have an important role in the early phase of follicular growth, before follicles become follicle-stimulating hormone (FSH) sensitive. They inhibit apoptosis in follicles and improve the action of FSH. In line with these findings, it has been reported that the use of androgen patches increased both pregnancy and take home baby rates in POR women $(9,10)$.

As apart from the decreased retrieval of mature oocytes, the decline in endometrial receptivity might adversely affect reproductive performance in aging women. Accordingly, the reality of endometrial aging and the critical role of androgens have been noted (11). Such as with ovarian aging, it is reasonable to assume a decline in efficiency of endometrium receptivity during the advancing reproductive period. It is most likely that androgenic endometrial milieu changes as women age because DHEA levels significantly decrease with advancing age $(1,2)$.

Some extragonadal tissues consist of steroidogenic enzymes that may convert DHEA to active androgens and estrogens. This cell-specific intracrinology may cause the local production of potent metabolites in accordance with cell-specific requisitions. In support, a regulatory effect of androgens on endometrial cell survival has been reported (12). In view of the above mentioned facts, we hypothesized that DHEA supplementation in POR women may improve reproductive performance through other mechanisms than the number of retrieved oocytes. The endometrium, therefore, may be the most likely area where potential positive effects of DHEA are seen. Although expression of androgen receptor (AR) has been reported in the human endometrium (13), the effect of DHEA on endometrium receptivity genes remained elusive. There is no controlled study investigating the possible effect of DHEA supplementation on homeobox genes (HOXA-10 and HOXA-11) and leukemia-inhibitory factor (LIF). These are the key receptivity genes that regulate decidualization and implantation rates $(14,15)$. To detect the possible influence of DHEA on receptivity we compared the expression intensities of endometrial HOXA-10, HOXA-11, and LIF mRNA in POR women who were given DHEA and others that were not.

\section{Material and Methods}

The primary outcome of this work was to investigate the hypothesis that DHEA improves endometrium receptivity in POR. The secondary outcome was to determine spontaneous clinical pregnancy rates. The presence of at least two of the following three features were accepted as a POR (16): (i) Maternal age $\geq 40$ years; (ii) Previous history of retrieving fewer than 3 oocytes; (iii) Previous history of decreased ovarian reserve [antral follicle count (AFC), 5-7 follicles or antimüllerian hormone $(\mathrm{AMH}), 0.5-1.1 \mathrm{ng} / \mathrm{mL}]$. Some women in our study had lower levels of AMH than $0.5 \mathrm{ng} / \mathrm{mL}$. Most of the POR patients in the study group were aged less than 40 years and met the criteria in sections (ii) and (iii).

Twenty-eight women with POR fulfilled the eligibility criteria and participated in the study. At the initial visit, although the participants had a diagnosis of POR, they underwent confirmatory ultrasound examination for their AFC. Blood was also obtained from all participants for AMH measurement. Following verbal informed consent and local Institutional Review Board approval, the POR women were equally divided in to two groups as subjects who were currently using DHEA $(n=14)$ and those were not $(n=14)$. POR women with a history of taking DHEA for at least 6 weeks were included in the DHEA treatment group. This time interval was chosen because of the early follicular growth induced by DHEA that occurs within 2 months of treatment. The POR women who were not using DHEA were accepted as the control group. Five fertile subjects were accepted as the second control group. POR women in the treatment group received oral DHEA with $25 \mathrm{mg} / \mathrm{TID}$. Women with untreated hydrosalpinges, submucous or intramural leiomyomas, endometrial polyps, male factor infertility, and tubal factor infertility were excluded. Women with endocrine disorders such as insulin-dependent diabetes mellitus, congenital adrenal hyperplasia, thyroid diseases, and hyperprolactinemia were also excluded. Women with a history of allergy to DHEA were not included. Endometrial thickness was measured at the late follicular phase following hysteroscopy and serum samples were obtained for hormonal evaluation.

In order to establish endometrial causes of former failed in vitro fertilization (IVF) cycles and local endometrial damage, the decision for hysteroscopy was taken for both groups of POR participants. All subjects underwent hysteroscopy at the late follicular phases and endometrial samples were obtained. Following repetitive washing of samples with a saline solution, they were transferred into an RNA stabilization buffer and stored at $-80{ }^{\circ} \mathrm{C}$. POR subjects were left for a $4-5$ month waiting period after hysteroscopy; if the women did not achieve pregnancy during this time period they underwent in vitro fertilization/ intracytoplasmic sperm injection (IVF/ICSI).

\section{Real-time polymerase chain reaction (RT-PCR)}

\section{Sample preparation and RNA isolation}

Both the RT-PCR method used for measuring expression levels of endometrial mRNA and comparative RNA 
expression analysis methods were the same as used in the recent study conducted by our team; more information can be found elsewhere (17). Unless otherwise specified, the kits used in this study were obtained from Qiagen, Hilden, Germany.

\section{Reverse transcription cDNA synthesis}

Complementary DNA was prepared by using a QuantiTect Reverse Transcription kit (17).

\section{RT-PCR analysis of homeobox and LIF genes}

Both positive controls and other genes were prepared using PrimerDesign to analyze the efficacy of the PCR reaction. The mRNA levels of sampling tissues were normalized to that of the house-keeping gene ( $\beta$-actin) mRNA level. RT-PCR results are expressed as cycle threshold $(\mathrm{Ct})$, delta $\mathrm{Ct}(\Delta \mathrm{Ct})$ and ddCt $(\Delta \Delta \mathrm{Ct})$. For the calculation of average $\mathrm{Ct}$ values, each endometrial sample was studied three times. The sequence and accession numbers of all primers designed to be used as forward and reverse primers for RT-PCR were: HOXA10(NM_018951),F-5'-GGTTTGTTCTGACTTTTTGTTTCT-3', R-5' T GACAC T TAGGACAATATCTATCTCTA-3'; HOXA11(NM_005523),F-5'-AGTTCTTTCTTCAGCGTCTACATT-3', R - 5' T T T T T C C T T C A T T C T C C T G T T C T G - 3'; LIF(NM_002309),F-5'GGAGGTCACTTGGCATTCAG-3', R-5'GGAAGAGAACGAAGAACCTACC-3'; andACTB(NM_001101), F-5'GCAAGCAGGAGTATGACGAGT-3', R-5'CAAGAAAGGGTGTA ACGCAACTAA-3'.

\section{Statistical analysis}

The expression of the studied genes was determined using the $2^{-\Delta \Delta C T}$ method. All data were normalized according to the ACTB gene ( $\beta$-actin) mRNA content. The normalized gene expression of POR women was divided by the normalized gene expression of the control subjects. The fold increase were considered positive or an up-regulation for transcript overexpression when the corresponding mRNA level was at least 2-fold higher than that of the initial transcript expression, negative or downregulation, if lower than 2 -fold. The Kolmogorov-Smirnov test showed normal distributions of data. The ANOVA test with post hoc Tukey's procedure and Mann-Whitney U test were used for analyzing continuous variables. Pearson's Chi-square test was used for analyzing other data. $\mathrm{P}<0.05$ was accepted as statistically significant. The results are given as mean and standard deviation (SD).

\section{Results}

POR women on DHEA and without DHEA had similar age, AFC, FSH, and endometrial thickness (Table 1). Significantly lower $\mathrm{AMH}$ levels were detected in women on DHEA compared with women who were not taking DHEA ( $0.33 \pm 0.23$ vs. $0.64 \pm 0.12 \mu \mathrm{g} /$ $\mathrm{mL})$. The mean age of the fertile controls was $33.1+2.3$ years. The mean age of the POR women on DHEA (33.0 \pm 4.57 years), those without DHEA (33.2 \pm 6.18 years), and controls were similar. The mean baseline FSH $(5.6 \pm 0.5 \mathrm{mIU} / \mathrm{mL})$ levels of the fertile group were lower than in POR women on DHEA $(10.8 \pm 1.12$ $\mathrm{mIU} / \mathrm{mL}$ ). The mean baseline endometrial thickness of the fertile group (7.4 \pm 0.5$)$ and women on DHEA (7.73 \pm 0.79$)$ were similar. The mean baseline AFCs of the fertile group (4.67 \pm 1.87 ) was higher than in POR women on DHEA (1.80 \pm 0.67$)$. Trends toward an increase in clinical spontaneous pregnancy rates in POR women on DHEA were detected compared with the POR women who were not given DHEA (53.3\% vs. 43.8\%). However, the difference was found insignificant $(\mathrm{p}<0.59)$. One subject had early pregnancy loss among the POR women on DHEA, and 3 of the 14 women who were not given DHEA had pregnancy loss (Table 1).

The expression levels of endometrial HOXA-10 and HOXA11 mRNA of subjects who were not given DHEA and fertile women were the same $(\mathrm{p}<0.44$ and $\mathrm{p}<0.25$ respectively).

Table 1. Clinical characteristics of POR women on DHEA and without DHEA

\begin{tabular}{|l|l|l|l|}
\hline & POR on DHEA & POR without DHEA & p* value \\
\hline Age (years) & $33.0 \pm 4.57$ & $33.2 \pm 6.18$ & 0.79 \\
\hline AMH (ng/mL) & $0.33 \pm 0.23$ & $0.64 \pm 0.12$ & $<0.0001$ \\
\hline AFC & $1.80 \pm 0.67$ & $2.00 \pm 0.63$ & 0.38 \\
\hline FSH (mIU/mL) & $10.8 \pm 1.12$ & $10.3 \pm 1.20$ & 0.29 \\
\hline Endometrial thickness (mm) & $7.73 \pm 0.79$ & $7.25 \pm 0.68$ & 0.07 \\
\hline Infertility duration (year) & $7.53 \pm 3.22$ & $6.10 \pm 3.76$ & 0.25 \\
\hline Previous IVF attempt & $1.00 \pm 1.30$ & $1.21 \pm 1.80$ & 0.93 \\
\hline Clinical pregnancy rates (\%) & 53.3 & 0.59 \\
\hline Early pregnancy loss & 1 & 3 & - \\
\hline $\begin{array}{l}\text { AMH: anti-müllerian hormone; AFC: antral follicle count; FSH: follicle-stimulating hormone; IVF: in vitro fertilization; POR: poor responders; Data are } \\
\text { presented as mean } \pm \text { standard deviation; *p<0.05 is accepted statistically significant }\end{array}$ & \\
\hline
\end{tabular}


Expression levels of LIF mRNA were found to be lower in the endometrium of POR subjects who were not given DHEA as compared with the fertile controls. However, the difference was noted as insignificant $(p<0.48)$. Upregulated HOXA-10 and 11 mRNA expressions were found in women taking DHEA. POR women on DHEA showed a 7.33-fold increment in HOXA-10 mRNA expression, and a 2.39-fold increment was detected in HOXA-11 mRNA expression. Only the increment in HOXA-10 mRNA expression was significant $(p<0.03)$. The fold increment in HOXA-11 mRNA after DHEA supplementation was found as 2.39, which was greater than two, thus indicating an upregulation. However, this increment was not significant $(\mathrm{p}<0.45)$. Likewise, an insignificant increase in LIF mRNA expression (1.76-fold, $\mathrm{p}<0.36$ ) was detected after DHEA supplementation. Due to the fold rise in LIF mRNA following DHEA being smaller than 2 -fold, it was considered to be down-regulation (Table 2, Figure 1).

口HOXA10 口HOXA11 口LIF

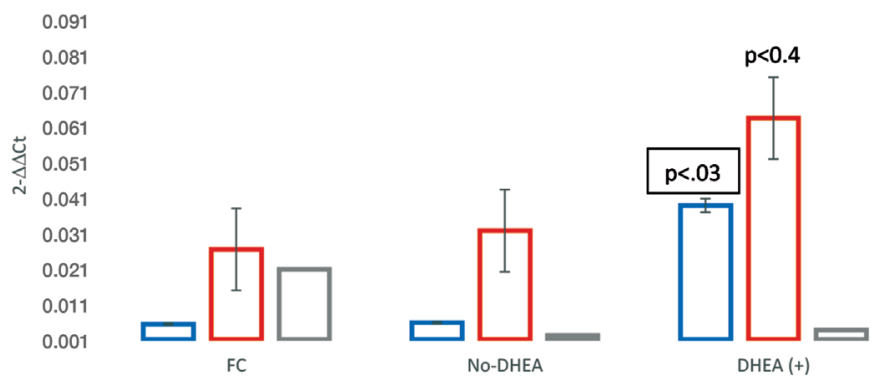

Figure 1. The relative gene expression was determined using the $2-\Delta \Delta \mathrm{CT}$ method. All data were compared with the fertile control group and normalized to ACTB gene (actin, beta) mRNA content. No-DHEA; POR women who were not given DHEA, DHEA (+); POR women on DHEA

FC; fertile control; $P O R$ : poor responders

\section{Discussion}

The two main results arising from this study are the increased endometrial HOXA-10 mRNA expression and the spontaneous pregnancy rates being higher than those reported in the literature. Although DHEA supplementation increases follicle recruitment, potentializes gonadotrophin effect, reduces follicle apoptosis, and enhances IGF-1 levels, how DHEA improves fertility outcomes is still not accurately known (18-20). Barad et al. (21) reported the clinical pregnancy rates in DOR women on DHEA as 10.9-28.1\%, and half of the pregnancies occurred spontaneously. In the current study, more than $50 \%$ of POR women on DHEA (53.3\%) conceived within 4-5 months after the hysteroscopy. In line with our results, a study conducted by Fusi et al. (22) showed that DHEA improved the chance of spontaneous pregnancies in POR women. High spontaneous pregnancy rates in POR patients on DHEA might be due to the positive impact of DHEA on the endometrial microenvironment, as well as the positive impact on oocyte development.

In order to clarify enhanced endometrial receptivity following DHEA administration, both average $2^{-\Delta C t}$ and fold change values for each gene were measured. The main result from the current study is the 7.3-fold rise in HOXA-10 and 2.3-fold rise in HOXA-11 mRNA expression after DHEA. Based on upregulated expressions of both genes, we can strongly suggest that exogenous DHEA improves endometrial receptivity. However, it is not evident whether DHEA has a direct effect on endometrial receptivity or its function only as a precursor to estrogens. Accordingly, androgens are not solely a substrate for estrogen production but may also modulate the effects of estrogen in the endometrium (23). If we accept the idea that DHEA increases endometrial receptivity by transforming into estrogen, it is logical to believe that administration of exogenous estrogens should improve endometrial receptivity. Confirmation of our hypothesis comes from an egg donation study of women with

Table 2. Comparison of expression levels of endometrial HOXA-10, HOXA-11, and LIF mRNA in the fertile control, POR women on DHEA, and without DHEA

\begin{tabular}{|c|c|c|c|c|c|c|}
\hline Gene & Group & Average $2-\Delta \mathrm{Ct}$ & Fold change & 95\% CI & $p$ value & Regulation \\
\hline \multirow{3}{*}{ HOXA-10 } & No-DHEA & 0.005799 & 1.0852 & $0.00001-2.54$ & 0.446 & -* \\
\hline & DHEA (+) & 0.03918 & $7.3322^{\mathrm{a}}$ & $0.00001-16.97$ & $0.032^{\mathrm{b}}$ & UP (7.3-fold) \\
\hline & Fertile control & $0.001-0.011$ & NA & - & - & - \\
\hline \multirow[t]{3}{*}{ HOXA-11 } & No-DHEA & 0.041521 & 1.5562 & $0.00001-4.02$ & 0.254 & $*$ \\
\hline & DHEA $(+)$ & 0.064035 & $2.3999^{a}$ & 0.00001-6.63 & 0.451 & UP (2.3- fold) \\
\hline & Fertile control & 0.025 & NA & - & - & - \\
\hline \multirow[t]{3}{*}{ LIF } & No-DHEA & 0.002173 & 1.038 & $0.00001-2.22$ & 0.480 & $*$ \\
\hline & DHEA (+) & 0.003692 & 1.7639 & $0.00001-5.91$ & 0.364 & $*$ \\
\hline & Fertile control & 0.021 & $\mathrm{NA}$ & - & - & - \\
\hline
\end{tabular}

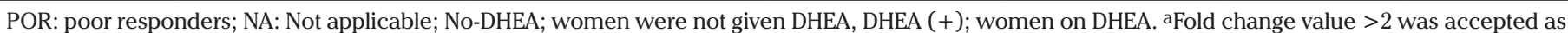
positive regulation for the genes studied. ${ }^{\mathrm{b}} \mathrm{p}<0.05 ;{ }^{*}$ No significant change was detected compared with controls 
advanced reproductive age (24). Estrogen priming of these women improves both endometrial thickness and implantation. However, increased implantation is not only due to the positive impact of estrogen. It should be remembered that good quality oocytes from healthy donors may overcome any age-related receptivity defect.

Peripheral interconversion of DHEA to active androgens, estrogens, and progesterone may be the first reason of the increased endometrial HOXA10 mRNA expression. It is a wellknown fact that expression of homeobox genes are modulated by sex steroids (25). Concordantly, endometrial HOXA10 mRNA was found to be associated with circulating 17- $\beta$ estradiol (25). Likewise, androgens are also regulators of the HOXA10 gene (26). As DHEA turns estrogens in peripheral tissues $(27,28)$, exogenous DHEA can exhibit a positive impact on endometrial HOXA10 mRNA expression in POR women on DHEA.

The second possibility of the positive effect of DHEA on HOXA10 mRNA might be the substitution of androgens or estrogens because circulating levels of androgen and estrogen decrease with advancing age (29). Nevertheless, local transformation of DHEA of other steroids in the endometrium is restricted by the altered levels of steroidogenic enzymes (11). Some authors believe in the local production of estradiol in the endometrium, whereas others do not support this notion $(30,31)$. The lack of aromatase activity and the existence of endometrial atrophy in postmenopausal women support the idea that the endometrium does not have the ability to produce local estrogen $(11,30)$. In contrast, Bukulmez et al. (32) reported that mRNA expression of aromatase enzyme in cultured endometrial cells were up-regulated by androstenedione (33). If a rise in HOXA10 mRNA following DHEA is secondary to conversion of DHEA to estrogens, why does endometrial thickness not alter significantly? In contrast to the stimulatory effect of estrogen on the endometrium, DHEA does not exert a stimulatory impact on endometrium (7). In good agreement with this, Labrie et al. (34) reported that the stimulatory impact of DHEA on the vagina was not detected in the endometrium of postmenopausal women and their endometrium remained atrophic after one year of DHEA supplementation. Likewise, endometrial thickness of women on DHEA and those that were not given DHEA were similar in our study. Together, upregulation of endometrial HOXA10 mRNA after DHEA treatment could be attributed to either DHEA itself or a function of its active compounds. If absolute androgen deficiency has a negative impact on endometrial androgen levels, we may suggest that exogenous DHEA may lead to a rise in local androgens that induce endometrial HOXA-10 mRNA expression.

AR have been shown in both pre- and postmenopausal human endometrium $(35,36)$. Estrogen increases AR, and progesterone inhibits it $(37,38)$. Therefore, a third possibility of augmented HOXA-10 expression after DHEA might be related to an AR enhancer effect of DHEA. The greatest support for our hypothesis come from the study by Qin et al. (39) who investigated the impact of DHEA on decidual PRL-related protein (dPRP), AR, and HOXA-10 expressions in mouse endometrial cells. They reported that DHEA had an insignificant effect on endometrial dPRP expression. The authors also noted that when given the dose of $100 \mathrm{nM}$, DHEA caused a significant increase in HOXA-10 mRNA. Moreover, they showed that DHEA-mediated upregulation in HOXA-10 was diminished by treatment with an AR antagonist. Together, if physiologic androgen deficiency in aging women leads to a decline in AR, we can suggest that exogenous DHEA can increase endometrial AR, which might lead to a rise in HOXA-10 (39).

The fourth possibility of improved receptivity after DHEA may be related to decidualization. By stimulating decidual prolactin production, androgens regulate decidualization $(40,41)$. Exogenous DHEA might upregulate HOXA-10 mRNA expression because homeobox genes induce decidualization and pinopode formation $(14,42)$.

There are some limitations to the current study. The study population is small in the studied groups. Alterations in mRNA expression are not confirmed by protein analyses. As opposed to our findings, patients with hyperandrogenism secondary to Polycystic Ovarian syndrome (PCOS) demonstrated low HOXA-10 and $\beta 3$-integrin expression $(43,44)$, suggesting androgens may have a detrimental impact on the endometrium. For this reason, one may suggest that the use of exogenous DHEA could impair the endometrial micro-milieu and that there is no need for androgen supplementation in POR women. Actually, DHEA may cause a PCO-like appearance in ovaries of POR women $(45,46)$. However, as exogenous DHEA does not exactly mimic clinical and biochemical features of genuine PCOS, results obtained from subjects with $\mathrm{PCO}$-like ovaries cannot be applied to POR women on DHEA.

Finally, we demonstrated for the first time that oral DHEA supplementation augments endometrial HOXA-10 mRNA expression. As well as the known possible positive effect on the count of oocytes retrieved, DHEA may increase implantation and pregnancy rates by modulating receptivity genes (17) or signal molecules (47). The receptivity enhancing effects of DHEA might be realized via transformation of DHEA to active metabolites (Figure 2). If DHEA indeed has a positive impact on endometrial receptivity, it can be used to enhance implantation rates in women with POR. Whatever the mechanism, the present study showed that DHEA exerted a positive effect on endometrial receptivity. If our results are supported by extensive studies, augmentation of endometrial receptivity with DHEA 


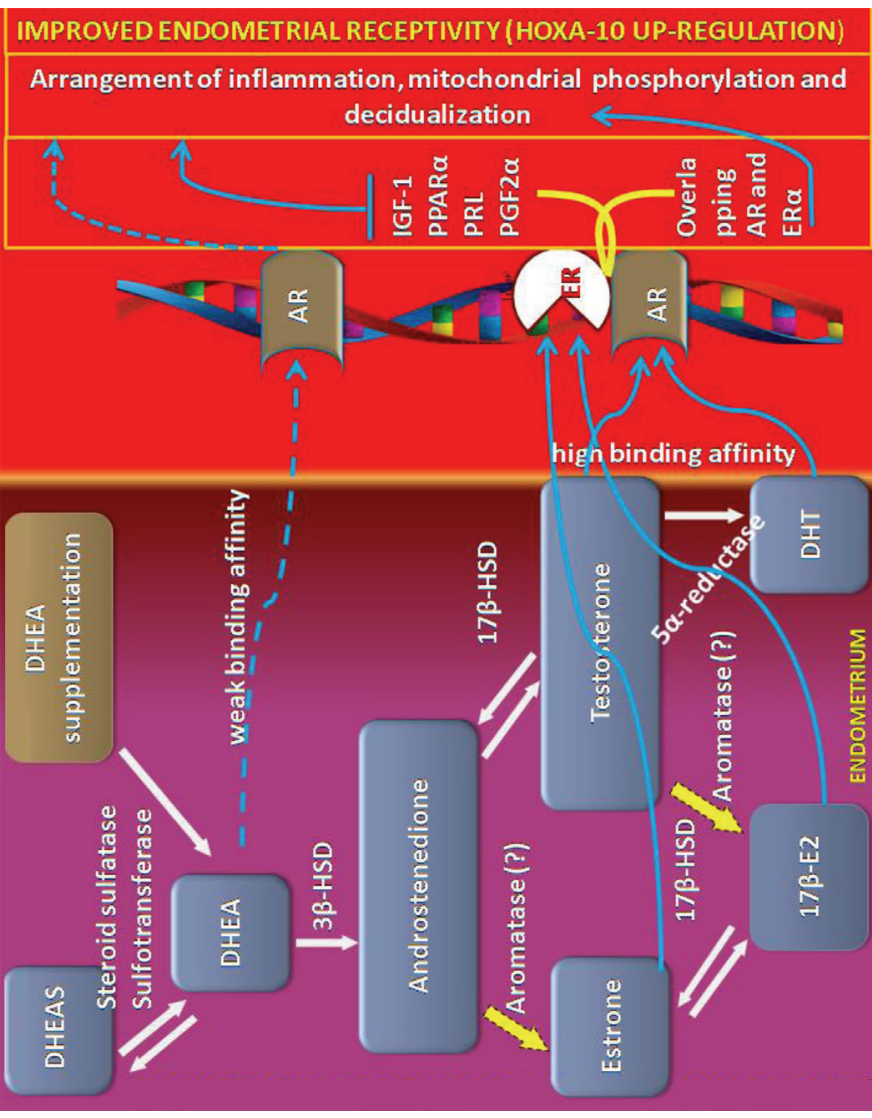

Figure 2. Abbreviated pathways to illustrate the possible mechanism of action of DHEA on endometrium receptivity.

might be a key factor for the management of women with implantation failure.

Acknowledgement: Authors acknowledge the help of Turkish German Gynecologic Research and Education Foundation for providing of HOXA and LIF genes kits.

Ethics Committee Approval: Ethics committee approval was received for this study from the ethics committee of Medipol University School of Medicine (No: 10840098-604.01.01E.13011).

Informed Consent: Verbal informed consent was obtained from patients who participated in this study.

Peer-review: Externally peer-reviewed.

Author Contributions: Concept - Ö.Ç., M.A.; Design - Ö.Ç., M.A., B.O., C.Ü., E.Ç.; Supervision - C.Ü., Ö.Ç., B.O., E.Ç.; Materials Ö.Ç., M.A., B.O., C.Ü., E.Ç.; Data Collection and/or Processing - A.I., N.Ç., A.E., L.H.A.; Analysis and/or Interpretation - B.O., Ö.Ç., M.A., C.Ü., E.Ç.; Literature Review - Ö.Ç., M.A., S.Ç.; Writer - Ö.Ç., M.A., B.O., C.Ü., E.Ç.; Critical Review - Ö.Ç., M.A., A.İ., N.Ç., A.E., L.H.A., B.O., S.Ç., E.Ç., C.Ü.
Conflict of interest: No conflict of interest is declared by the authors.

Financial Disclosure: The authors declared that this study received financial support from Turkish German Gynecologic Research and Education Foundation for HOXA and LIF genes kits.

\section{References}

1. Gellersen B, Brosens IA, Brosens JJ. Decidualization of the human endometrium: mechanisms, functions, and clinical perspectives. Semin Reprod Med 2007; 25: 445-53.

2. Erel CT, Aydin Y, Kaleli S, Ilvan S, Senturk LM, Is endometrial apoptosis evidence of endometrial aging in unexplained infertility? a preliminary report. Eur J Obstet Gynecol Reprod Biol 2005; 121: 195-201.

3. Labrie F, Luu-The V, Labrie C, Belanger A, Simard J, Lin SX, et al. Endocrine and intracrine sources of androgens in women: inhibition of breast cancer and other roles of androgens and their precursor dehydroepiandrosterone. Endocr Rev 2003; 24: 152-82.

4. Hillier SG, Whitelaw PF, Smyth CD. Follicular oestrogen synthesis: the 'two-cell, two-gonadotrophin' model revisited. Mol Cell Endocrinol 1994; 100: 51-4.

5. Ford JH. Reduced quality and accelerated follicle loss with female reproductive aging - does decline in theca dehydroepiandrosterone (DHEA) underlie the problem? J Biomed Sci 2013; 20: 93.

6. Labrie F, Martel C, Balser J. Wide distribution of the serum dehydroepiandrosterone and sex steroid levels in postmenopausal women: role of the ovary? Menopause 2011; 18: 30-43.

7. Labrie F, Bélanger A, Cusan L, Gomez JL, Candas B. Marked decline in serum concentrations of adrenal $\mathrm{C} 19$ sex steroid precursors and conjugated androgen metabolites during aging. J Clin Endocrinol Metab 1997; 82: 2396-402.

8. Hummer L, Nielsen MD, Christiansen C. An easy and reliable radioimmunoassay of serum androstenedione: age-related normal values in 252 females aged 2 to 70 years. Scand J Clin Lab Invest 1983; 43: 301-6.

9. Bosdou JK, Venetis CA, Kolibianakis EM, Toulis KA, Goulis DG, Zepiridis $\mathrm{L}$, et al. The use of androgens or androgen-modulating agents in poor responders undergoing in vitro fertilization: a systematic review and meta-analysis. Hum Reprod Update 2012; 18: 127-45.

10. Sunkara SK, Coomarasamy A. Androgen pretreatment in poor responders undergoing controlled ovarian stimulation and in vitro fertilization treatment. Fertil Steril 2011; 95: 73-4.

11. Labrie F, Labrie C. DHEA and intracrinology at menopause, a positive choice for evolution of the human species. Climacteric 2013; 16: 205-13.

12. Marshall E, Lowrey J, MacPherson S, Maybin JA, Collins F, Critchley $\mathrm{HO}$, et al. In silico analysis identifies a novel role for androgens in the regulation of human endometrial apoptosis. J Clin Endocrinol Metab 2011; 96: 1746-55.

13. Carneiro MM, Morsch DM, Camargos AF, Reis FM, Spritzer PM. Androgen receptor and 5alpha-reductase are expressed in pelvic endometriosis. BJOG 2008; 115: 113-7.

14. Lim H, Ma L, Ma WG, Maas RL, Dey SK. Hoxa-10 regulates uterine stromal cell responsiveness to progesterone during implantation and decidualization in the mouse. Mol Endocrinol 1999; 13: 1005-17.

15. Senturk LM, Arici A. Leukemia inhibitory factor in human reproduction. Am J Reprod Immunol 1998; 39: 144-51.

16. Ferraretti AP, La Marca A, Fauser BC, Tarlatzis B, Nargund G, Gianaroli L, et al. ESHRE consensus on the definition of 'poor response' to ovarian stimulation for in vitro fertilization: the Bologna criteria. Hum Reprod 2011; 26: 1616-24. 
17. Celik O, Unlu C, Otlu B, Celik N, Caliskan E. Laparoscopic endometrioma resection increases peri-implantation endometrial HOXA-10 and HOXA-11 mRNA expression. Fertil Steril 2015; 104: 356-65.

18. Gold EB, Sternfeld B, Kelsey JL, Brown C, Mouton C, Reame N, et al. Relation of demographic and lifestyle factors to symptoms in a multi-racial/ethnic population of women 40-55 years of age. Am J Epidemiol 2000; 152: 463-73.

19. Casson PR, Lindsay MS, Pisarska MD, Carson SA, Buster JE. Dehydroepiandrosterone supplementation augments ovarian stimulation in poor responders: a case series. Hum Reprod 2000; 15: 2129-32.

20. Casson PR, Santoro N, Elkind-Hirsch K, Carson SA, Hornsby PJ, Abraham G, et al. Postmenopausal dehydroepiandrosterone administration increases free insulin-like growth factor-I and decreases high-density lipoprotein: a six-month trial. Fertil Steril 1998; 70: 107-10.

21. Barad D, Brill H, Gleicher N. Update on the use of dehydroepiandrosterone supplementation among women with diminished ovarian function. J Assist Reprod Genet 2007; 24: 62934.

22. Fusi FM, Ferrario M, Bosisio C, Arnoldi M, Zanga L. DHEA supplementation positively affects spontaneous pregnancies in women with diminished ovarian function. Gynecol Endocrinol 2013; 29: 940-3.

23. Kowalski AA, Vale-Cruz DS, Simmen FA, Simmen RC. Uterine androgen receptors: roles in estrogen-mediated gene expression and DNA synthesis. Biol Reprod 2004; 70: 1349-57.

24. Klein J, Sauer MV. Oocyte donation. Best Pract Res Clin Obstet Gynaecol 2002; 16: 277-91.

25. Taylor HS, Arici A, Olive D, Igarashi P. HOXA10 is expressed in response to sex steroids at the time of implantation in the human endometrium. J Clin Invest 1998; 101: 1379-84.

26. Zanatta A, Rocha AM, Carvalho FM, Pereira RM, Taylor HS, Motta EL, et al. The role of the Hoxa10/HOXA10 gene in the etiology of endometriosis and its related infertility: a review. J Assist Reprod Genet 2010; 27: 701-10

27. Barad D, Gleicher N. Effect of dehydroepiandrosterone on oocyte and embryo yields, embryo grade and cell number in IVF. Hum Reprod 2006; 21: 2845-9.

28. Labrie F. DHEA after menopause - sole source of sex steroids and potential sex steroid defi ciency treatment. Menopause Management 2010; 19: 14-24.

29. Gleicher N, Kim A, Weghofer A, Kushnir VA, Shohat-Tal A, Lazzaroni $\mathrm{E}$, et al. Hypoandrogenism in association with diminished functional ovarian reserve. Hum Reprod 2013; 28: 1084-91

30. Labrie F. All sex steroids are made intracellularly in peripheral tissues by the mechanisms of intracrinology after menopause. $\mathrm{J}$ Steroid Biochem Mol Biol 2015; 145: 133-8.

31. Bulun SE, Lin Z, Imir G, Amin S, Demura M, Yilmaz B, et al. Regulation of aromatase expression in estrogen-responsive breast and uterine disease: from bench to treatment. Pharmacol Rev $2005 ;$ 57: 359-83.
32. Bukulmez O, Hardy DB, Carr BR, Auchus RJ, Toloubeydokhti T, Word RA, et al. Androstenedione up-regulation of endometrial aromatase expression via local conversion to estrogen: potential relevance to the pathogenesis of endometriosis. J Clin Endocrinol Metab 2008; 93: 3471-7.

33. Hausknecht V, Lopez de la Osa E, Gurpide E. In vitro metabolism of C19 steroids in human endometrium. J Steroid Biochem 1982; 17: 621-9.

34. Labrie F, Diamond P, Cusan L, Gomez JL, Bélanger A, Candas B. Effect of 12-month dehydroepiandrosterone replacement therapy on bone, vagina, and endometrium in postmenopausal women. J ClinEndocrinol Metab 1997; 82: 3498-505.

35. Horie K, Takakura K, Imai K, Liao S, Mori T. Immunohistochemical localization of androgen receptor in the human endometrium, decidua, placenta and pathological conditions of the endometrium. Hum Reprod 1992; 7: 1461-6.

36. Jones MC, Fusi L, Higham JH, Abdel-Hafiz H, Horwitz KB, Lam EW, et al. Regulation of the SUMO pathway sensitizes differentiating human endometrial stromal cells to progesterone. Proc Natl Acad Sci U S A 2006; 103: 16272-7.

37. Kalantaridou SN, Davis SR, Nelson LM. Premature ovarian failure. Endocrinol Metab Clin North Am 1998; 27: 989-1006.

38. Kang Z, Pirskanen A, Janne OA, Palvimo JJ. Involvement of proteasome in the dynamic assembly of the androgen receptor transcription complex. J Biol Chem 2002; 277: 48366-71.

39. Qin A, Qin J, Jin Y, Xie W, Fan L, Jiang L, et al. DHEA improves the antioxidant capacity of endometrial stromal cells and improves endometrium receptivity via androgen receptor. Eur J Obstet Gynecol Reprod Biol 2016; 198: 120-6.

40. Zhang X, Croy BA. Maintenance of decidual cell reaction by androgens in the mouse. Biol Reprod 1996; 55: 519-24.

41. Narukawa S, Kanzaki H, Inoue T, Imai K, Higuchi T, Hatayama H, et al. Androgens induce prolactin production by human endometrial stromal cells in vitro. J Clin Endocrinol Metab 1994; 78: 165-8.

42. Nikas G, Aghajanova L. Endometrial pinopodes: some more understanding on human implantation? Reprod Biomed Online 2002; 4: 18-23.

43. Cermik D, Selam B, Taylor HS. Regulation of HOXA-10 expression by testosterone in vitro and in the endometrium of patients with polycystic ovary syndrome. J Clin Endocrinol Metab 2003; 88: 238-43.

44. Thornton JW. Evolution of vertebrate steroid receptors from an ancestral estrogen receptor by ligand exploitation and serial genome expansions. Proc Natl Acad Sci U S A 2001; 98: 5671-6.

45. Luchetti CG, Solano ME, Sander V, Arcos ML, Gonzalez C, Di Girolamo $\mathrm{G}$, et al. Effects of dehydroepiandrosterone on ovarian cystogenesis and immune function. J Reprod Immunol 2004; 64: 59-74.

46. Barad DH, Gleicher N. Increased oocyte production after treatment with dehydroepiandrosterone. Fertil Steril 2005; 84: 756.

47. Ersahin A, Celik O, Acet M, Ersahin S, Acet T, Bozkurt DK, et al. Impact of Endometrioma Resection on Eutopic Endometrium Metabolite Contents: Noninvasive Evaluation of Endometrium Receptivity. Reprod Sci 2016; 24: 795-5. 